\title{
Malignant Peritoneal Mesothelioma Following Wilms' Tumor in a Horse-Shoe Kidney, A Case Report and Review of Literature
}

Ahmed S Abdulsamad ${ }^{1}$, Ayesha Inam ${ }^{1}$, Muharrem Oner $^{1}$, Humaid 0 Al-shamsi ${ }^{3}$, Sadir J Alrawi ${ }^{2}$, Thamir Alkasab, Tamer Madi ${ }^{1}$ and Humaa Darr ${ }^{1}$

${ }^{1}$ Surgical Oncology Fellow, Oncology Department, Al-Zahra Cancer Center, UAE

${ }^{2}$ Head of Oncology Department, Al-Zahra Cancer Center, UAE

${ }^{3}$ Consultant Medical Oncologist, Al Zahra Cancer Center, UAE

Submission: February 27, 2019; Published: March 21, 2019

"Correspondence Author: Ahmed S Abdulsamad, Surgical Oncology Fellow, Oncology Department, Al-Zahra Cancer Center, UAE

\begin{abstract}
In this case report we are presenting a 50 years old lady with a history of nephroblastoma resection at the age of 4 and then presented with a vague abdominal discomfort that was diagnosed as a malignant peritoneal mesothelioma, treated by peritonectomy, cytoreductive surgery with HIPEC and post-operative chemotherapy. In this review we are trying to address the connection between the 2 neoplasms genetically and environmentally.
\end{abstract}

Keywords: Peritoneal mesothelioma; Wilms' tumor; Nephroblastoma; Horse shoe kidney; HIPEC

\section{Introduction}

Wilms' tumour (WT) or nephroblastoma is a relatively rare disease of childhood, with about 70 children per year developing WT compared with approximately 1,500 children a year who develop cancer under the age of 15 years in the UK. WT mostly affects children below the age of 5 [1]. Only 1-3\% have a family history of the disease, with inheritance appearing to be autosomal dominant with incomplete penetrance and linkage studies show evidence for multiple familial WT genes [2,3].

Approximately $2 \%$ occur as part of recognized syndromes: the WAGR (WT, aniridia, genitourinary malformation and mental retardation) syndrome and Denys-Drash syndrome are due to germline mutations in the WT1 gene. Beckwith Widemann syndrome is associated with a variety of genetic abnormalities at the $11 \mathrm{p} 15$ locus. WT also occurs in association with isolated hemi hypertrophy or genitourinary malformations, though the genes involved are currently unidentified $[4,5]$. The development of second primary neoplasms (SPNs) in survivors of childhood cancer is one of the more serious late effects, and these often occur many years after the diagnosis of the original cancer $[6,7]$. There is a significantly increased risk of mesothelioma and renal cell carcinoma in a Wilms' tumor affected population, and both rare tumors have been shown to be increased after irradiation in survivors of childhood cancer and of WT $[8,9]$.

Several neoplasms appear to be overrepresented in individuals with Malignant Mesothelioma (MM), including some recognized as part of the BAP1 tumor predisposition syndrome or described in BAP1 mutation carriers. The reasons for the higher than expected prevalence of non-mesothelial neoplasms not associated with BAP1 mutations in individuals with MM requires further exploration. Because the occurrence of nonmesothelial neoplasms in individuals with MM is uncommon, BAP1 mutational testing may be beneficial in such individuals to better elucidate the spectrum of neoplasms that arise in the setting of BAP1 mutations [10].

\section{Case Report}

This is a 50 years old lady presented with a vague abdominal pain at the hypogastric and right iliac fossa region that started in November 2017. The patient had some mild abdominal discomfort of insidious onset with gradually progressive course. Her surgical history started when she was 1 year old, she was diagnosed as having recurrent attacks of urinary tract infections 


\section{Cancer Therapy \& Oncology International Journal}

due to bilateral vesicoureteric reflux and a horse shoe kidney, for which she underwent bilateral implantation of ureters into the urinary bladder. At the age of 4 , she was diagnosed as having Wilms' tumor of the lower pole of left kidney that was being treated by conservative surgery followed by chemotherapy and external beam radiation; the pathological examination came to reveal Nephroblastoma.

Unfortunately, this irradiation did destroy her both ovaries. When she turned 15, she was diagnosed as having gonadal dysfunction, scanty axillary and pubic hair, no breast development and primary amenorrhea. Karyotyping done revealing normal 46XX chromosomes. She had hormonal replacement therapy starting at the age of 15, taking Estinyl and adopted one child. In 2011, she had total abdominal hysterectomy because of multiple uterine fibroids and heavy bleeding, leaving both ovaries without being touched. This was followed by bilateral oophorectomy done on April 2015 that showed chronic endosalpingiosis in the right side and the left side shows chronic inflammation.

CT scan of the Abdomen and Pelvis with IV and oral contrast was done in Jan 2018 for that pain, it showed homogenous enhancement of the pancreas associated with $12 \times 13 \mathrm{~mm}$ cyst seen in the body with no septations nor mural nodules and without any enhancement, there was also an enhancing nodule seen involving left adrenal gland measuring $8 \times 7 \mathrm{~mm}$ in size. Kidneys are connected in the lower pole (horse shoe kidney anomaly) with malrotation of the renal pelvis and a mild degree of pelvicalyceal fullness (Figures $1 \& 2$ ).

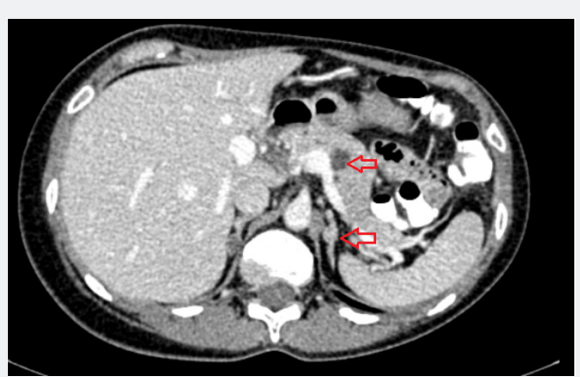

Figure 1:CT scan of the abdomen with IV and oral contrast, axial view at $L 1 / L 2$ level showing left adrenal nodule, cyst $12 * 13$ $\mathrm{mm}$ at the body of the pancreas.

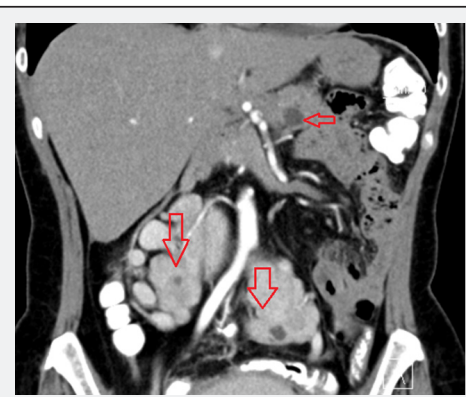

Figure 2:CT scan of the abdomen with IV and oral contrast, Coronal reconstruction showing the Horse-shoe kidney (note the decreased volume of left kidney due to previous resection of left lower pole, benign looking pancreatic body cyst.
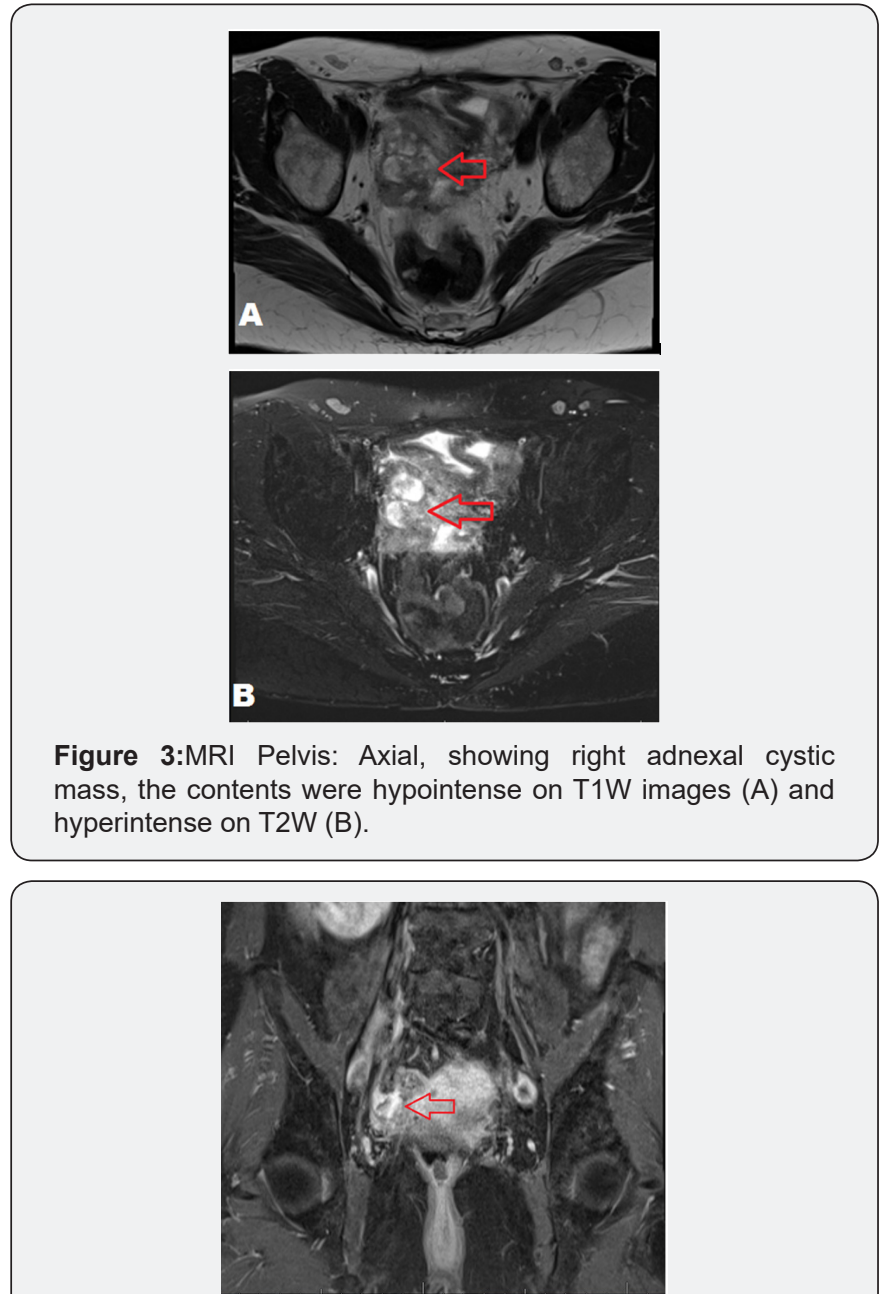

Figure 4:MRI Pelvis, Coronal view showing the right adnexal cysts with contrast enhancement.

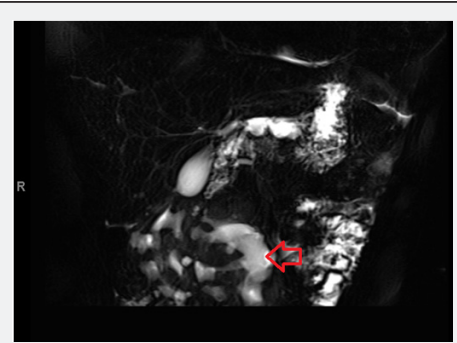

Figure 5:MRI abdomen T2W, Showing the Horse-shoe Kidney (arrow)

Tumor markers done at that time, CA 19-9 was 8, Following up CA 125, it kept rising from $190 \mathrm{U} / \mathrm{ml}$ in December 2017, to $1225 \mathrm{U} / \mathrm{ml}$ in September 2018 to $1438 \mathrm{U} / \mathrm{ml}$ in October 2018. Due to the elevation of CA 125 level, MRI Pelvis was done in Feb 2018 Pre- and post-contrast multiplanar imaging of the pelvis including a dynamic sequential post-contrast study done revealing multiple cysts seen in right adnexa measuring $9.5 \times 14.7 \times 16.5 \mathrm{~mm}$ and $11.2 \times 11.3 \times 11.7 \mathrm{~mm}$. The contents were hyperintense on T2W and hypointense on T1W images. Smooth enhancement of the wall was seen on post contrast study. Fluid 


\section{Cancer Therapy \& Oncology International Journal}

was seen in pelvis as well (Figures 3-5). PET scan was done in August 2018 which showed peritoneal stranding and nodular hypermetabolic soft tissue nodule anterior to the transverse colon. Soft tissue thickening posterior to the right aspect of the urinary bladder. It was not conclusive!

Due to the over-rising CA125, she was subjected to laparoscopic exploration, omental, peritoneal, biopsies, peritoneal fluid cytology on 4th of Oct-2018, histological sections from peritoneum, mesocolon, and momentum, peritoneal cytology showed similar features of atypical cells infiltrating fibroadipose tissue. Immunostain results came as: neoplastic cells are strongly positive for Calretinin and EMA, show weak focal positivity for D2-40 and moderate degree of p53 overexpression. Neoplastic cells were completely negative for Ber-EP4 and PAX8. Concluded an interpretation of malignant mesothelioma of peritoneum- poorly differentiated.

This case was discussed in the tumor board at Al-zahra cancer center Dubai -UAE, the board committee agreed to go ahead with Cytoreductive surgery, peritonectomy, HIPEC procedure. The patient had surgery done in October 2018; Cystoscopy was done with ascending nephrogram and bilateral ureteric stents were inserted (Figure 6). Midline abdominal incision was done, after careful dissection, lysis of adhesions; Formal exploratory laparotomy has been entertained.

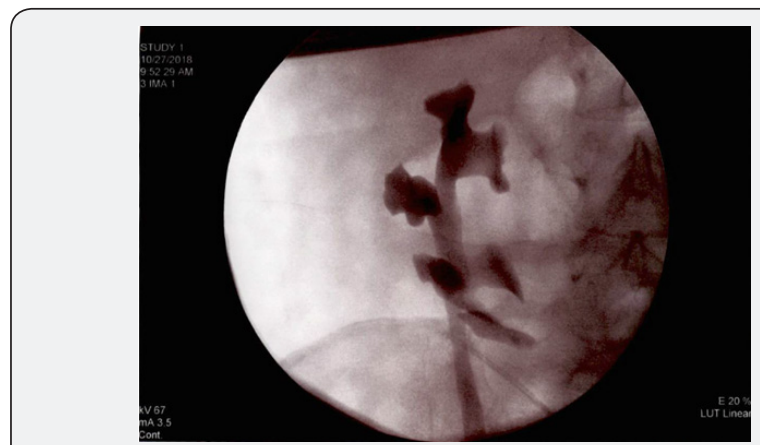

Figure 6:Ascending Nephrogram, intraoperatively, showing medially directed pelvicalyceal system of right kidney.

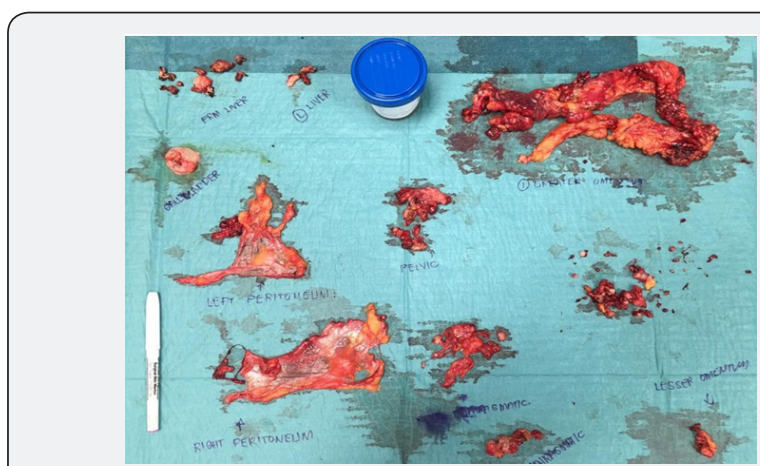

Figure 7: Specimens post Cytoreductive surgery \& peritonectomy, note the resected nodules from the small and large bowel, parietal peritoneum, pelvic peritoneum, gall bladder, hepatic nodules.
From the DJ flexure, we ran the bowel, most of the implants were removed and sent for pathological examination ,frozen section biopsy came as Peritoneal nodule positive for poorly differentiated malignancy, and for deposits non amenable for resection; argon beamed, we continued in that direction until we ended into the terminal ileum, the appendix could not be identified. The right colon, transverse colon, and the splenic flexure were mobilized. Completion rigid sigmoidoscopy was done that showed no evidence of any bleeding nor air leak. Greater and lesser omentectomy, cholecystectomy was entertained, peritoneal carcinoma index was more than 20 (Figure 7).

Formal peritonectomy (Parietal, diaphragmatic, Pelvic) done and sent for pathology evaluation (R0). Washing of the peritoneal cavity, putting the tunneled catheters for HIPEC and closing the abdomen temporarily. Then the heated intraperitoneal chemoperfusion started. Protocol has been written by our medical oncologists, double dose in the first hour and half-dose in the second hour of Mitomycin C. After that we took all the fluids out, standard chemo disposal was performed with good irrigation, two Jackson-Pratt drains, size 19 were left behind and size 15 in the subcutaneous tissue followed by closure of the wound in layers. Abdominal binder was applied. The patient tolerated the procedure very well and was sent to the ICU for post-operative follow up.

Histological sections of the entire specimen showed multiple nodules which are formed by malignant epitheloid proliferation with infiltrative growth pattern. Neoplastic cells were papillary, tubular, trabecular, and nested growth pattern. They showed moderate degree of pleomorphism; however, mild rare mitosis also identified. Cells had large nucleus with prominent nucleoli and abundant pink cytoplasm. Some of the cells also show mesothelial like fimbriae. Gallbladder mucosa appears unremarkable; however, a small foci of tumor cell is noted on the serosal surface, Ki-67 proliferative index was $15-20 \%$.

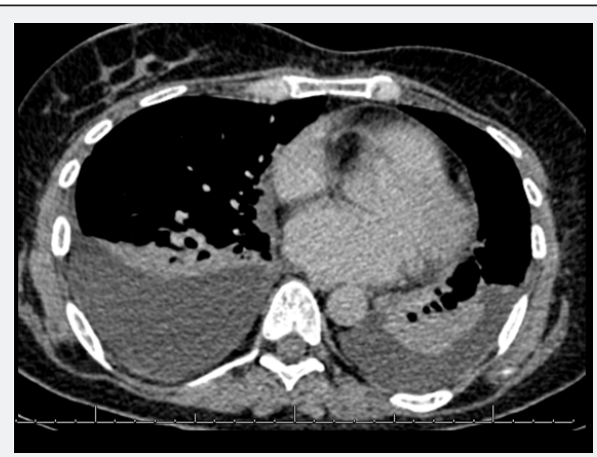

Figure 8: Plain Axial CT scan of the chest, showing bilateral pleural effusion, more on the right side, post Cytoreductive surgery and HIPEC.

According to these criteria, 6 cycles of adjuvant chemotherapy were decided including cisplatin and pemetrexed. During the post-operative period in ICU, patient had a right sided pleural 
effusion, with satisfactory oxygen saturation in room air, that's why we conservatively managed this effusion that resolved completely in days, this raises a question whether a chest tube should be inserted routinely at the end of HIPEC for drainage of a potential Right pleural effusion (Figure 8). Post CRS and HIPEC, CA 125 went down to $361 \mathrm{U} / \mathrm{ml}$ on November 2018.

\section{Discussion}

Diffuse malignant peritoneal mesothelioma (DMPM) has been traditionally regarded as a rapidly lethal disease. Recently, several independent prospective trials have reported improved survival with an intensive loco-regional treatment strategy including cytoreductive surgery (CRS) along with peri-operative intra-peritoneal chemotherapy (PIC). However, most of the surgical data comes from mono-institutional phase I or II studies and there is a broad range of variability regarding inclusion criteria, cytoreductive surgical procedures, drugs, temperatures and methods of delivering the heated chemotherapy [11].

Plones and colleagues have addressed the connection between malignant pleural mesothelioma and nephroblastoma as follows: The Wilms Tumor Protein 1 (WT1) is a transcription factor which is highly expressed by MPM and is involved in cellular development and survival. They evaluated the role of WT1 in two human MPM cell lines (MSTO and H2052) expressing high levels of WT1. They performed a knockdown of WT1 using siRNA which was confirmed by Westernblotting. After knockdown of WT1 they investigated the effect on proliferation, chemoresistance, chemotaxis and migration. They could demonstrate that knockdown of WT1 suppresses chemoresistance in both cell lines compared with control (scrambled siRNA). Additionally, WT1 knockdown reduces proliferation, chemotaxis and invasiveness of mesothelioma cell lines. WT1 reduces malignancy of malignant mesothelioma cell lines and might be a new molecular target in mesothelioma therapy [12].

In a study done by Jean S. Lee et al. [13] 2,851 patients were diagnosed with Wilms' tumor as their first malignancy. Of these, 34 patients were reported to have a secondary malignant neoplasms (SMN). Cumulative incidence for a secondary malignancy was $0.6 \%$ (95\% confidence interval [95\% CI] $0.3-$ $1.0 \%$ ) at 10 years, $1.6 \%(95 \%$ CI $1.0-2.3 \%)$ at 20 years, and $3.8 \%$ (95\% CI $2.4-5.9 \%$ ) at 30 years. Median time from primary diagnosis to SMN diagnosis was 12.5 years. standardized incidence ratio for SMN for survivors of Wilms' tumor was 3.4 (95\% CI 2.2-4.9) with an absolute excess risk of 7.6 per 10,000 persons per year. Exposure to radiation did not significantly increase risk for development of second malignancy. Overall survival for patients with SMN was $64.5 \%$ at 5 years [13].

Another study by Anna Scattone and colleagues included Histological samples of 31 malignant peritoneal mesothelioma were stained immunohistochemically for the WT1 protein. The results were quantified by recording the number of stained nuclei, and then correlated with patient survival. Statistical correlation was evaluated for tumour histotype, mitotic count (MC), nuclear grade (NG), necrosis, lymphoid response (grade of inflammation) and desmoplasia with regard to survival. Highgrade histology (solid epithelioid, pure sarcomatoid or biphasic tumours), high NG, MC more than five per 10 per high-power field (HPF), necrosis and desmoplasia were associated with a significantly worse prognosis. Patients with MPM with low WT1 expression ( $\leq 25 \%$ of positive cells) survived for a significantly shorter time compared to those with high WT1 expression ( $>25 \%$ of positive cells) $(\mathrm{P}=0.0001)$. The $50 \%$ survival time of subjects with low WT1 expression was 2.9 months [95\% confidence interval (CI): 2.05-3.71] versus 31.5 months (95\% CI: 20.4-42.5) for those with high WT1 expression. On multivariate analysis, WT1 and MC were found to be associated independently with survival $(\mathrm{P}=0.002 ; \mathrm{P}=0.005$, respectively). suggesting that low WT1 expression and high MC may be indicative of an unfavorable prognosis in patients with advanced malignant peritoneal mesothelioma [14].

In a prospective study by Marcello et al, patients with PM were treated by cytoreductive surgery (CRS) and intraperitoneal hyperthermic perfusion (IPHP). Mean follow-up was 27 months (range: 1-65). Fifteen (68\%) patients had malignant disease, two had well-differentiated papillary mesothelioma, and two had multicystic PM. Thirteen (65\%) patients received preoperative chemotherapy. Fifteen cases (75\%) underwent optimal cytoreduction (residual disease $<2.5 \mathrm{~mm}$ ). One patient underwent the procedure twice due to locoregional progression. IPHP was performed with closed abdomen technique, using a preheated polysaline perfusate $\left(42.5^{\circ} \mathrm{C}\right)$ containing cisplatin + mitomycin $\mathrm{C}$ or cisplatin + doxorubicin administered through a heart-lung pump for 60 or $90 \mathrm{~min}$ [15].

Three-year overall and progression-free survival was 69 and $66 \%$, respectively. The operative morbidity (grade II/ III), mortality, and overall toxicity (grade I-IV) rates were 25, 0 , and $30 \%$, respectively. Seventeen (94\%) out of 18 patients had resolution of ascites [15]. A database of one of the authors consisting of $3900 \mathrm{MM}$ cases received for professional and medicolegal consultation from 1982 through 2016 was searched retrospectively for individuals with MM who also had a history of one or more antecedent or synchronous non-mesothelial neoplasms.16 There was a history of prior irradiation of a non-mesothelial malignancy in 33 individuals with MM, most commonly Hodgkin lymphoma (16 of 19 cases), breast carcinoma ( 8 of 17 cases), and Wilms' tumor ( 2 of 2 cases). These 33 individuals represented $14 \%$ of all individuals in the study population who had both MM and a non-mesothelial neoplasm [16].

BAP1 tumor predisposition syndrome is characterized by autosomal dominant germline mutations in BAP1 [17]. The function of this gene is not entirely understood, but the BAP1 protein is a deubiquitinating enzyme involved in key cellular 


\section{Cancer Therapy \& Oncology International Journal}

processes, including tumor suppression [18]. Recognized components of the BAP1 tumor predisposition syndrome (Online Mendelian Inheritance in Man [OMIM] 614327) [19] include atypical Spitz tumors, cutaneous and ocular (uveal) melanoma, malignant mesothelioma (MM), lung carcinoma, clear cell renal cell carcinoma, and meningioma. An array of other tumors has been reported in BAP1 mutation carriers, including breast cancer and pleomorphic undifferentiated sarcoma [17].

Malignant mesothelioma has also been reported to arise following therapeutic radiation of an antecedent malignancy and is likely a contributing factor in at least some of the cases in our study population $[20,21]$.

They observed an increased prevalence of various types of non-mesothelial malignancies typically treated with irradiation in individuals with MM. Details regarding some of these individuals have been previously published [20]. Although the timing and field of radiation was not available in all cases, several non-mesothelial neoplasms occurred and were treated with radiation years earlier with MM subsequently arising in the field of radiation.

One recent study [22] that did examine the risk of second malignancies in individuals with MM from a population-based perspective found an association with kidney cancer but not with other cancers associated with the BAP1 tumor predisposition syndrome. Other studies that have examined the risk of second neoplasms using SEER data include several focused specifically on uveal or cutaneous melanoma, which did not demonstrate a substantially increased risk of subsequent MM. A limitation of these studies is that the follow-up period may not have adequately captured the latency period associated with MM [23, 24].

\section{Conclusion}

Malignant peritoneal mesothelioma can arise as a metachronous malignant tumor post Wilms' tumor in a period up to 40 years, predisposed either genetically or related to irradiation in the childhood or a combination of both, better understanding of BAP1 tumor predisposition syndrome need to be clearly studied in a prospective study in a large sample.

\section{Conflicts of Interest}

The authors declare that there is no conflict of interest regarding the publication of this article.

\section{References}

1. Childhood Cancer (2004) Factsheet. Cancer Research UK.

2. Pritchard-Jones K, Hawkins MM (1997) Biology of Wilms' tumour. Lancet 349: 663-664.

3. Hawkins MM, Winter DL, Burton HS, Potok MHN (1995) Heritability of Wilms' tumor. J Natl Cancer Inst 87: 1323.

4. Pritchard-Jones K, Vujanic G (2006) Multiple pathways to Wilms tumor: how much is genetic? Pediatr Blood Canc 47: 232-234.
5. Breslow NE, Beckwith JB, Perlman EJ, Reeve AE (2006) Age distributions, birth weights, nephrogenic rests and heterogeneity in the pathogenesis of Wilms tumor. Pediatr Blood Canc 47(3): 260-267.

6. Paulino AC, Wen BC, Brown CK, Tannous R, Mayr NA (200) Late effects in children treated with radiation therapy for Wilms' tumor. Int J Radiat Oncol Biol Phys 46(5): 1239-1246.

7. Breslow NE, Takashima JR, Whitton JA, Moksness J, D'Angio GJ, et al. (1995) Second malignant neoplasms following treatment for Wilms' tumour: a report from the National Wilms' Tumor Study Group. J Clin Oncol 13(8): 1851-1859.

8. Pappo AS, Santana VM, Furman WL, Kun LE, Walter AW, et al. (1997) Post-Irradiation malignant mesothelioma. Cancer 77(7):1379-85.

9. Cherullo EE, Ross J, Kay R, Novick A (2001) Renal neoplasms in adult survivors of childhood. J Urol 165(1): 2013-2017.

10. Kelly J Butnor, Elizabeth N Pavlisko, Thomas A Sporn, Victor L Roggli, et al. (2018) Malignant Mesothelioma in Individuals with Nonmesothelial Neoplasms. Arch Pathol Lab Med 142 (6): 730-734

11. Marcello Deraco, David Bartlett, Shigeki Kusamura, Dario Baratti (2008) Consensus statement on peritoneal mesothelioma. J. Surg. Oncol. Wiley-Liss, Inc. 98(4): 268-272.

12. Plönes, Fischer, M, Höhne, K. et al. (2017) Turning back the Wheel: Inducing Mesenchymal to Epithelial Transition via Wilms Tumor 1 Knockdown in Human Mesothelioma Cell Lines to Influence Proliferation, Invasiveness, and Chemotaxis. Pathol Oncol Res Springer Netherlands 23(4): 723-730.

13. Jean S Lee, Benjamin Padilla, Steven G DuBois, Aris Oates, John Boscardin (2015) Second malignant neoplasms among children, adolescents and young adults with Wilms tumor. Pediatric Blood \& Cancer. Wiley Periodicals 62 (7): 1259-1264.

14. Scattone A, Serio G, Marzullo A, Nazzaro P, Corsi F, et al. (2012) High Wilms' tumour gene (WT1) expression and low mitotic count are independent predictors of survival in diffuse peritoneal mesothelioma. Blackwell publishing limited. Histopathology 60(3): 472-481.

15. Marcello Deraco, Paolo Casali, MG Inglese, Dario Baratti, Elisabetta Pennacchioli, et al. (2003) Peritoneal mesothelioma treated by induction chemotherapy, cytoreductive surgery, and intraperitoneal hyperthermic perfusion. J. Surg. Oncol. Wiley-Liss, Inc 83(3): 147-153.

16. Kelly J Butnor, Elizabeth N Pavlisko, Thomas A Sporn, Victor L Roggli (2018) Malignant Mesothelioma in Individuals with Nonmesothelial Neoplasms. Arch Pathol Lab Med 142(6): 730-734.

17. Carbone M, Yang H, Pass HI, Krausz T, Testa JR, et al. (2013) BAP1 and cancer. Nat Rev Cancer 13(3): 153-159.

18. Pilarski R, Rai K, Cebulla C, Abdel-Rahman M (1993) BAP1 tumor predisposition syndrome. In: Pagon RA, Adam MP, Ardinger HH, et al, eds. GeneReviews. Seattle: University of Washington, USA.

19. Online Mendelian Inheritance in Man (2014) OMIM. Baltimore, MD: Johns Hopkins University, USA.

20. Goodman JE, Nascarella MA, Valberg PA (2009) Ionizing radiation: a risk factor for mesothelioma. Cancer Causes Control 20(8): 12371254.

21. Li X, Brownlee NA, Sporn TA, Mahar A, Roggli VL (2015) Malignant (diffuse) mesothelioma in patients with hematologic malignancies a clinicopathologic study of 45 cases. Arch Pathol Lab Med 139(9): 1129-1136.

22. Chen T, Kharazmi E, Lou J, Zhang X, Sundquist K, et al. (2016) Risk of second primary cancers after malignant mesothelioma and vice versa. Cancer Lett 379(1): 94-99. 


\section{Cancer Therapy \& Oncology International Journal}

23. Lains I, Bartosch C, Mondim V, et al. (2016) Second primary neoplasms in patients with uveal melanoma: a SEER database analysis. Am J Ophthalmol 165: 54-64.
24. Spanogle JP, Clarke CA, Aroner S, Swetter SM (2010) Risk of second primary malignancies following cutaneous melanoma diagnosis: a population-based study. J Am Acad Dermatol 62(5): 757-767.

\section{Your next submission with Juniper Publishers} will reach you the below assets

- Quality Editorial service

- Swift Peer Review

- Reprints availability

- E-prints Service

- Manuscript Podcast for convenient understanding

- Global attainment for your research

- Manuscript accessibility in different formats ( Pdf, E-pub, Full Text, Audio)

- Unceasing customer service

Track the below URL for one-step submission https://juniperpublishers.com/online-submission.php 